\title{
Recombination line profiles of embedded clusters (Research Note)
}

\author{
S. C. Beck \\ Department of Physics and Astronomy, Tel Aviv University, Ramat Aviv, Israel \\ e-mail: sara@wise1.tau.ac.il \\ Received 23 June 2008 / Accepted 1 August 2008 \\ ABSTRACT

\begin{abstract}
We are trying to probe conditions in the youngest super star clusters, those still embedded in dense obscuring clouds. The hydrogen recombination lines in the radio and infrared can be observed through the obscuration, as the optical and UV lines cannot, and give us the kinematics of the ionized gas. The line profiles of the clusters resemble superpositions of the lines of many very young ultracompact or hyper-compact HII regions. This can be explained if each OB star is individually embedded in dense material which it is accreting, even as it ionizes. We speculate on what this implies for conditions in the clusters.
\end{abstract}

Key words. galaxies: star clusters - infrared: general - line: profiles

\section{Introduction}

Compact, populous star clusters are the main mode of star formation in many starburst galaxies. These Super Star Clusters (SSCs) contain a few $10^{5}$ to a few $10^{7} M_{\odot}$ in radii 3 to $10 \mathrm{pc}$. (Turner \& Beck 2003; Johnson 2004; Tsai et al. 2006; Gilbert \& Graham 2007, and references therein.) SSCs are expected to contribute greatly to the mechanical energy, mass transport and metal enrichment of the starburst. In their mature stages, when they may be measured directly, they are observed to drive strong winds that can create superbubbles and strongly influence the galactic ISM (Tenorio-Tagle et al. 2007). The evolution of these cluster winds is fairly well understood and the models agree reasonably well with what is observed (Silich et al. 2007).

But the very youngest clusters, still deeply embedded in gas and dust clouds, are not so well understood. They cannot be observed in the optical or UV because of the extinction, but only at infrared, millimeter and radio wavelengths. The cluster stars are not directly observable; only the nebula they excite can be measured. These cluster sources are called RISN (radio-infrared supernebulae) or UDHII (ultra-dense HII regions) (Turner \& Beck 2003; Kobulnicky \& Johnson 1999). They contain as many as $10000 \mathrm{O}$ stars in volumes no larger than a few parsecs (Turner \& Beck 2004, 2003; Henry et al. 2007; Beck et al. 2002), yet do not have the cluster winds of their more evolved counterparts. The gas motions in these clusters, as revealed in infrared and radio recombination line shapes, do not resemble O star winds. In this note we discuss the line shapes and what they imply for the internal structure and evolution of the nebula and cluster.

\section{Observations}

The RISN of embedded star clusters differ from the HII regions associated with evolved SSCs. The HII regions of SSCs are usually more extensive than the stellar component. The sizes of RISN are hard to measure. The only one which has clearly been resolved is $0.7 \times 0.9$ pc (Turner \& Beck 2004); for others, the size is estimated by comparing the observed radio brightness temperature $T_{\mathrm{b}}$ and the electron temperature $T_{\mathrm{e}}$ and is usually found to be 1-5 pc diameter. The stars in RISN have not been observed directly except possibly in NGC 5253 (Turner \& Beck 2004) but the radio sources are so small that the stars cannot reasonably be confined to an even smaller voume. So for RISN sources, the nebular gas and the stars are coextensive.

The high mid-infrared fluxes of RISN argue that they are associated with a lot of hot dust. There is dust mixed throughout the nebulae, not outside them in obscuring shells; this is demonstrated in the dependance of the measured extinction on the wavelength. The total optical depth $\left(A_{\mathrm{v}}\right)$ to an RISN derived from the ratio of optical $\mathrm{H} \alpha$ and $\mathrm{H} \beta$ Balmer lines is typically much lower than that derived from the infrared $\operatorname{Br} \alpha$ and $\operatorname{Br} \gamma$ lines. In NGC 5253 the optical lines give $A_{\mathrm{v}}$ of $3 \mathrm{mag}$, the infrared 18 mag (Turner et al. 2003). In II Zw 40 the Balmer lines give $0.2 \mathrm{mag} A_{\mathrm{v}}$ and the Brackett lines about 8-10 mag (Beck et al. 2002), similar to Henize 2-10 (Henry et al. 2007). The extinction derived from the ratio of thermal radio emission to $\operatorname{Br} \alpha$ is usually even higher than that found from two Brackett lines. This means that the emitters and absorbers are mixed, so the depth to which an observation can see depends on the wavelength of the observation, and the longer wavelengths see deeper into the source. (Note that if the measured extinction at $\lambda, A_{\lambda}$, is greater than $1 \mathrm{mag}$, it implies that observations at $\lambda$ do not see through the source; this is the case for Balmer lines towards most RISN.)

\subsection{Observations of line shapes}

The kinematics of the RISN are best studied through the infrared recombination lines $\operatorname{Br} \alpha$ and $\operatorname{Br} \gamma$. These can be measured with better spatial resolution than radio recombination lines, and while not free of extinction, can usually see well into the RISN. We have acquired $\operatorname{Br} \alpha$ and $\operatorname{Br} \gamma$ spectra of RISN in many nearby starbursts using the NIRSPEC spectrometer on the Keck telescope. The spectra of He 2-10, NGC 5253 and II Zw 40 have been published (Henry et al. 2007; Turner et al. 2003, 2001) 
Table 1. RISN line shapes and characteristics.

\begin{tabular}{cccccc}
\hline \hline Source & O stars & $\begin{array}{c}M_{\text {tot }} \\
10^{6} M_{\odot}\end{array}$ & $\begin{array}{c}\text { Radius } \\
\mathrm{pc}\end{array}$ & $\begin{array}{c}F W H M \\
\left(\mathrm{~km} \mathrm{~s}^{-1}\right)\end{array}$ & $\begin{array}{c}F W Z I \\
\left(\mathrm{~km} \mathrm{~s}^{-1}\right)\end{array}$ \\
\hline NGC 5253 & 1200 & 1.0 & $0.7 \times 0.9$ & 76 & no broad component \\
II Zw 40 & 600 & 0.1 & 0.5 & 103 & 270 \\
He 2-10 1 & 1900 & 1.3 & $0.4-1$ & $57-78$ & $220-330$ \\
He 2-10 2 & 1100 & 0.7 & $0.4-1$ & $57-78$ & $22-330$ \\
He 2-10 4N & 2300 & 1.5 & $0.4-1$ & $57-78$ & $220-330$ \\
He 2-10 4S & 2400 & 1.7 & $0.4-1$ & $57-78$ & $220-330$ \\
He 2-10 5 & 1200 & 0.8 & $0.4-1$ & $57-78$ & $220-330$ \\
M 83 & 530 & 0.2 & - & 78 & no broad component \\
\hline
\end{tabular}

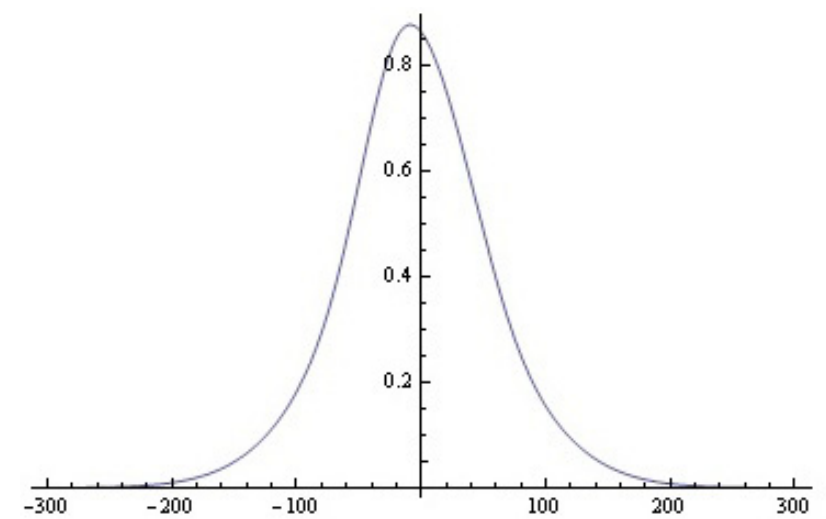

Fig. 1. Simulated line shapes for a cluster of 30 UCHII regions, as described in the text.

and M 83 is in preparation (Crosthwaite et al. 2008). Gilbert \& Graham (2007) have Br $\gamma$ spectra of many rather less embedded sources in the Antennae galaxies.

The FWHM of the RISN lines observed ranges from less than 20 to over $100 \mathrm{~km} \mathrm{~s}^{-1}$, but the large majority fall in the range 50 to $100 \mathrm{~km} \mathrm{~s}^{-1}$. Some RISN, like that in NGC 5253, appear to have only one velocity component, and some (those in $\mathrm{He}$ 2-10 for example) have a weak and broad pedestal of emission with FWHM 250 to $550 \mathrm{~km} \mathrm{~s}^{-1}$. Typical RISN line shapes are shown in Turner et al. (2003), Henry et al. (2007), and Crosthwaite et al. (2008).

\section{RISN lines and UCHII region lines}

The RISN lines are remarkably narrow, considering that the sources contain at least many hundreds of $\mathrm{O}$ stars and that $\mathrm{O}$ stars have strong winds at velocities of many hundreds of $\mathrm{km} \mathrm{s}^{-1}$. The lines do not look like O star winds, even less like Wolf-Rayet star winds; what they resemble most are the lines seen from Galactic Ultra-Compact and Hyper-Compact HII (UCHII and HCHII) regions (a point made by Gilbert \& Graham (2007) as well). These Galactic sources are deeply embedded, excited by one or at most a few OB stars, and have infrared line widths in the same range of $F W H M$ as do the RISN (Garay \& Linzano 1999).

We were motivated by the similarity of line shapes to simulate the profiles that would be produced by the superposition of many UCHII region lines. This supposes a simple physical model in which each OB star is in its own UCHII region and the winds do not interact with each other. We neglect stars of lower masses, which while certainly present in the forming cluster, are not strongly ionizing and are not expected to influence the recombination line profiles. In Fig. 1, we show the result of superposing 30 Gaussian line shapes, each with central velocity

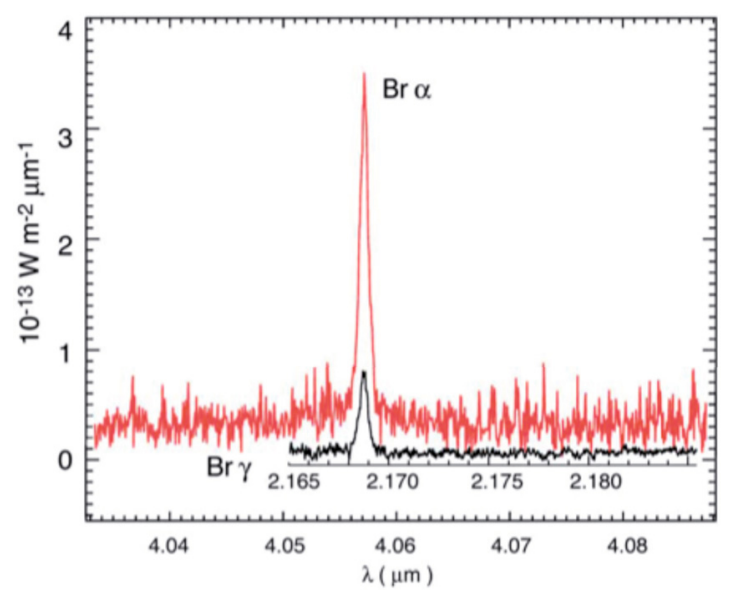

Fig. 2. The Brackett line profiles of the RISN in NGC 5253, from Turner et al. (2003). The small $(0.001 \mu \mathrm{m})$ ticks equal $74 \mathrm{~km} \mathrm{~s}^{-1}$. This RISN has only the narrow component.

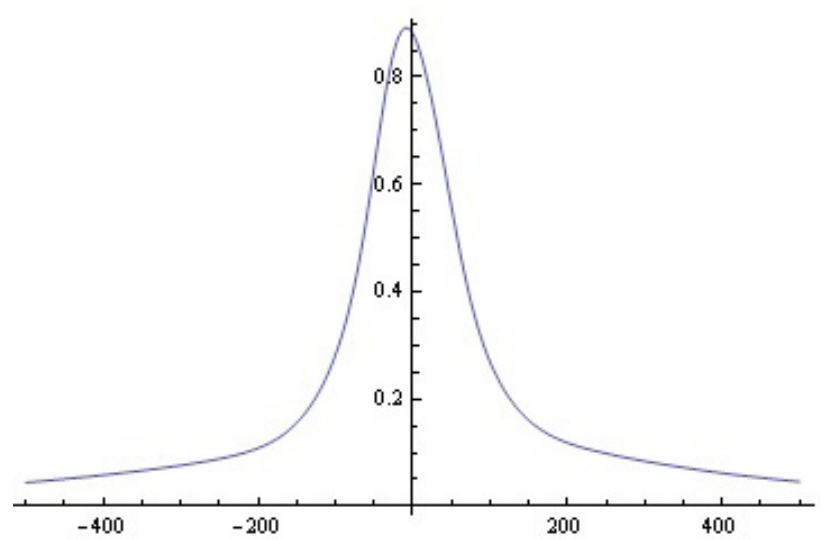

Fig. 3. Simulated line shapes for a cluster of 30 UCHII regions and 5 WR stars, as described in the text.

of a random number in the range -10 to $10 \mathrm{~km} \mathrm{~s}^{-1}$ and $F W H M$ of a random number in the range 30 to $100 \mathrm{~km} \mathrm{~s}^{-1}$. The spread in the allowed central velocity was chosen, since the stars in RISN cannot be observed directly, to match stellar velocity dispersions observed in visible super star clusters and globular clusters. Since the clusters embedded in RISN have masses similar to those of these visible clusters, this should be a fair assumption. The FWHM agrees with what is seen in UCHII regions. (It is not established that UCHII region lines are Gaussians, but the results do not depend on shape). In Fig. 2, we show the line profiles from the RISN in NGC 5253, a source with a narrow line and no wide component. Figure 3 has a simulated profile made of 30 random lines generated as above, with the addition of 5 sources whose central velocities were chosen in the same range but whose $F W H M$ was selected between 200 and $500 \mathrm{~km} \mathrm{~s}^{-1}$. This models a collection of young stars, some of which have begun to develop higher velocity stellar winds; the fast component is a typical line shape for a young star which is obscured but not in a UCHII region. Figure 4 shows the profiles of two RISN in He 2-10 which have broad pedestals in their lines. It may be seen that the shapes of the lines in Figs. 1 and 2, and 3 and 4, agree. 


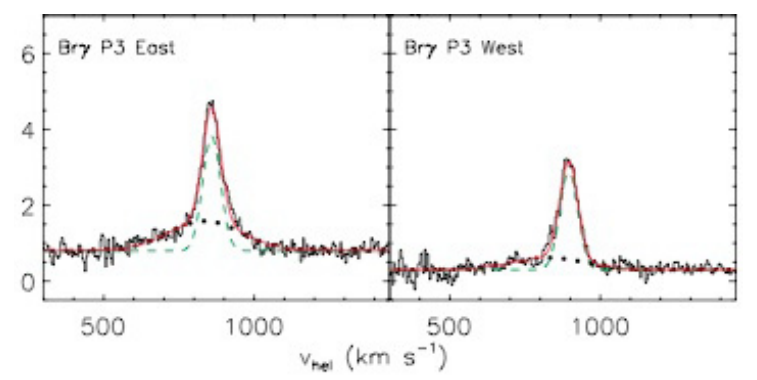

Fig. 4. Brackett line profiles of two RISN in He 2-10, from Henry et al. (2007). Intensity is relative to peak. These have both narrow and wide components.

\section{Implications for the structure of the nebula}

The line shapes generated by superposing UCHII region winds of randomly chosen central velocity and FWHM agree very well with the RISN line shapes; those with only low FWHM winds in the sum look like the NGC 5253 wind, and those with the higher velocity winds look like the sources in He 2-10. A difference is that the simulated lines have asymmetries from adding a relatively small number of sources whose central velocities differ by a significant fraction of their FWHM. This effect is expected to decrease as more sources are included. The observed asymmetries in the wide component of the lines in He 2-10 was not random but took the sense that the red wing of $\operatorname{Br} \gamma$, but not $\operatorname{Br} \alpha$, was weakened; probably an extinction effect.

That the RISN line shapes agree with those from a simulation of superimposed UCHII regions does not prove that the RISN actually is a superposition of UCHII regions. But it strongly suggests a relation, and that we can try to apply what we know of UCHII regions to the as-yet unobservable volume inside RISN. So what can we speculate about the internal structure of the RISN from the agreement with UCHII region line shapes? The current models for UCHII regions (Keto 2007; Hollenbach et al. 1993; Churchwell 2002) agree that the winds in these sources start while the star is still embedded in dense material and surrounded by a disk, that material continues to accrete onto the disk (and possibly elsewhere) in the early stages of the wind, and that this dense material suppresses and brakes the wind. As the surrounding material in the disk and the cloud is removed by accretion, radiation or wind pressure, the wind becomes faster and covers more solid angle. So dense ambient material is needed to maintain the wind in its UCHII configuration: in this model each OB star in the RISN is still embedded in its own nugget of obscuring material. This local material, which is in addition to any large shells of dust surrounding the entire cluster, is presumably responsible for the extinction pattern discussed above.
If the speculation that RISN contain many UCHII regions is correct, the evolutionary state of the RISN may relate to the line shapes as follows. In the very youngest all the $\mathrm{O}$ stars are deeply embedded and their winds suppressed so the net line is narrow, like that of NGC 5253. As some stars break out of the obscuration and produce faster winds the lines look like those of He 2-10, and when all the stars are freed of their cocoons, the RISN cluster will take on the appearance of a normal super star cluster and will develop a normal cluster wind.

\section{Conclusions}

We have demonstrated that the recombination line shapes observed in RISN are like those that would be seen from a cluster of UCHII regions. Since the line shapes of UCHII regions depend on dense obscuring material local to the star, this implies that the cluster are still embedded in, and accreting from, their individual cocoons. We suggest that in RISN whose lines show wider velocity components, some of the stars have removed their surrounding material and developed faster winds. Eventually, all the stars will have expelled their natal cocoons and the cluster will appear as a normal SSC.

Acknowledgements. We are grateful to Drs. Mike Shull and John Bally for discussions and hospitality, and to the referee for thoughtful comments.

\section{References}

Beck, S. C., Turner, J. L., Langland-Shula, L., et al. 2002, AJ, 124, 2516 Chevalier, R. A., \& Clegg, A. W. 1985, Nature, 317, 44

Churchwell, E. 2002, ARA\&A, 40, 27

Crosthwaite, L. P., Turner, J. L., Meier, D., \& Beck, S. 2008, ApJ, in press

Garay, G., \& Lizano, S. 1999, PASP, 111, 1049

Gilbert, A., \& Graham, J. 2007, ApJ, 668, 168

Henry, A., Turner, J. L., Beck, S. C., Crosthwaite, L. P., \& Meier, D. S. 2007, AJ, 133, 757

Hollenbach, D., Johnstone, D., \& Shu, F. 1993, ASP Conf. Ser. 35, ed. J. Cassinelli, \& E. Churchwell

Johnson, K. 2004, New Astron. Rev., 48, 1337

Keto, E., Zhang, Q., \& Kurtz, S. [arXiv:0708.3388v1]

Keto, E. 2007, ApJ, 666, 976

Kobulnicky, H., \& Johnson, K., ApJ, 527, 154

Silich, S., Tenorio-Tagle, G., \& Munoz-Tunon, C. 2007, ApJ, 669, 952

Tenorio-Tagle, G., Silich, S., \& Munoz-Tunon, C. 2007, NewAR, 51, 125

Tolstoy, E., \& Labhardt, L. 2003, ApJ, 590, 256

Tsai, C. W., Turner, J. L., Beck, S. C., et al. 2006, AJ, 132, 2383

Turner, J. L., \& Beck, S. C. 2003, in Star Formation at High Angular Resolution, ed. Jayawardhana, et al., ASP Conf. Ser., 221

Turner, J. L., \& Beck, S. C. 2004, ApJ, 602, L85

Turner, J. L., Beck, S. C., Crosthwaite, L. P., \& Meier, D. M. 2001, BAAS, 3, 1449

Turner, J. L., Beck, S. C., Crosthwaite, L. P., et al. 2003, Nature, 423, 621 